\title{
Genotype-phenotype correlation in a cohort of Portuguese patients comprising the entire spectrum of VWD types: impact of NGS
}

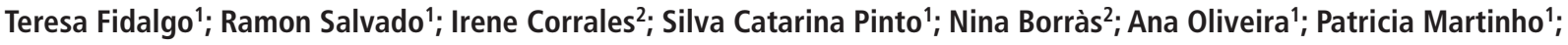 \\ Gisela Ferreira'; Helena Almeida'; Cristina Oliveira'; Dalila Marques'; Elsa Gonçalves'; MJoão Diniz ${ }^{3}$; Margarida Antunes ${ }^{3}$; \\ Alice Tavares³; Gonçalo Caetano4; Paula Kjöllerström"; Raquel Maia5; Teresa Sevivas'; Francisco Vidal²; Leticia Ribeiro \\ ${ }^{1}$ Centro Hospitalar e Universitário de Coimbra (CHUC) - Serviço de Hematologia Clínica, Unidade de Trombose e Hemostase, Hospital Pediátrico de Coimbra, Coimbra, Portugal; \\ ${ }^{2}$ Banc de Sang i Teixits (BST) - Unitat de Diagnòstic i Teràpia Molecular, Barcelona, Spain; ${ }^{3}$ Centro Hospitalar Lisboa Norte (CHLN) - Hospital de Santa Maria - Serviço de \\ Imunohemoterapia, Lisboa, Portugal; ${ }^{4}$ Hospital de Faro - Serviço Hematologia, Faro, Portugal; ${ }^{5}$ Centro Hospitalar Lisboa Central (CHLC) - Hospital D. Estefânia - Hematologia \\ Pediátrica, Lisboa, Portugal
}

\begin{abstract}
Summary
The diagnosis of von Willebrand disease (VWD), the most common inherited bleeding disorder, is characterised by a variable bleeding tendency and heterogeneous laboratory phenotype. The sequencing of the entire VWF coding region has not yet become a routine practice in diagnostic laboratories owing to its high costs. Nevertheless, nextgeneration sequencing (NGS) has emerged as an alternative to overcome this limitation. We aimed to determine the correlation of genotype and phenotype in 92 Portuguese individuals from 60 unrelated families with VWD; therefore, we directly sequenced VWF. We compared the classical Sanger sequencing approach and NGS to assess the value-added effect on the analysis of the mutation distribution in different types of VWD. Sixty-two different VWF mutations were identified, 27 of which had not been previously described. NGS detected 26 additional mutations, contributing to a broad overview of the mutant
\end{abstract}

\section{Correspondence to:}

Teresa Fidalgo

Centro Hospitalar e Universitário de Coimbra (CHUC)

Serviço de Hematologia Clínica

Unidade de Trombose e Hemostase

Av Afonso Romão Coimbra 3000-602, Portugal

Tel.: +351 239480 370, Fax: +351 239717216

E-mail: teresa.fidalgo@chuc.min-saude.pt

Supplementary Material to this article is available online at

www.thrombosis-online.com. alleles present in each VWD type. Twenty-nine probands (48.3\%) had two or more mutations; in addition, mutations with pleiotropic effects were detected, and NGS allowed an appropriate classification for seven of them. Furthermore, the differential diagnosis between VWD $2 B$ and platelet type VWD $(n=1)$, Bernard-Soulier syndrome and VWD $2 B(n=1)$, and mild haemophilia $A$ and VWD $2 N(n=2)$ was possible. NGS provided an efficient laboratory workflow for analysing VWF. These findings in our cohort of Portuguese patients support the proposal that improving VWD diagnosis strategies will enhance clinical and laboratory approaches, allowing to establish the most appropriate treatment for each patient.

\section{Keywords}

Gene mutations, von Willebrand disease, von Willebrand factor, molecular biology methods, NGS

Received: July 30, 2015

Accepted after major revision: March 2, 2016

Epub ahead of print: March 17, 2016

http://dx.doi.org/10.1160/TH15-07-0604

Thromb Haemost 2016; 116: 17-31

\section{Introduction}

Von Willebrand disease (VWD) is the most common inherited bleeding disorder, with a reported incidence of $0.01-1 \%(1,2)$. This disease is caused by a deficiency of von Willebrand factor (VWF), an adhesive glycoprotein of large dimensions with crucial functions in haemostasis derived from its ability to organise in multimers. The high-molecular-weight (HMW) multimers of VWF are essential for primary haemostasis, i.e. mediating a molecular endothelium-platelet bridge for binding collagen and the platelet receptors glycoprotein (GP)Ib and GPIIb/IIIa. In addition, VWF binds and stabilises factor VIII (FVIII) in the circulation, protecting it from rapid proteolytic degradation while delivering it to sites of vascular damage. As a result of this physiological pro- cess, VWF insufficiencies may cause haemorrhage by reducing platelet function or by reducing the FVIII concentration (1-3).

The diagnosis of VWD is based on the nature of the VWF deficiency. VWD is classified into three different types: partial or complete VWF quantitative deficiencies (types 1 and 3, respectively) and qualitative deficiency (type 2). Type 2 VWD is characterised by functional defects that lead to impaired activity and is accordingly divided into four secondary categories $(2 \mathrm{~A}, 2 \mathrm{~B}, 2 \mathrm{M}$ and $2 \mathrm{~N})$. Functional defects lead to enhanced (2B) or reduced (2A, 2M) platelet interaction or impaired binding to FVIII $(2 \mathrm{~N})$; structural defects due to impaired dimerisation/multimerisation can lead to type 2A/IID, 2A/IIC and 2A/IIE VWD $(1,4)$. Type 2A VWD can also be caused by mutations that impair the production of the larger multimers (Group I) and those with normal synthesis and 
release but with increased proteolytic susceptibility to ADAMTS13 (Group II) (5). All these defects can be identified by laboratory tests that assess the functional characteristics of VWF. It is crucial to correctly diagnose the subtype of VWD because the therapeutic approaches are different for each subtype. However, this diagnosis remains difficult because the clinical and laboratory phenotypes are very heterogeneous, and a clear discrimination between some of these subtypes can be challenging $(6,7)$.

Molecular analysis of VWF is very useful in the diagnosis and classification of VWD; type 2 mutations are localised to specific functional domains in exons 18-25 (type $2 \mathrm{~N}$ ), exon 28 (types $2 \mathrm{~A}$, $2 \mathrm{~B}$ and $2 \mathrm{M}$ ) and exons $11-16,24-26$ and $51-52$ (type $2 \mathrm{~A}$ ), whereas the type 1 and type $3 \mathrm{VWD}$ mutations require analysis of all the essential VWF regions. Moreover, compound heterozygosity for $V W F$ mutations in different functional domains of the VWF molecule causes pleiotropic effects and produces different phenotypes $(8,9)$.

To elucidate the clinical variability, which is found even in the same family, it is necessary to identify the mutational profile; however, the large size of VWF has been a disadvantage for studying using conventional Sanger DNA sequencing in diagnostic laboratories owing to its high costs. Nevertheless, the prospects for molecular diagnosis will be improved with the introduction of nextgeneration sequencing (NGS) platforms; massively parallel sequencing has reduced the cost and increased the throughput of DNA sequencing $(10,11)$.

With this in mind, we conducted a study with two main objectives: first, to better understand the genotype-phenotype correlation, we detailed the phenotypes of 92 Portuguese patients from 60 unrelated families with different types of VWD and VWF mutations; second, to decide the most appropriate strategy for VWD diagnosis in our Department of Haematology, including feasibility and costs, we compared the results obtained using conventional Sanger sequencing and NGS. This approach allowed the creation of a new algorithm that uses an efficient and cheaper methodology to establish the diagnosis, prognosis and a more accurate treatment for VWD.

\section{Materials and methods \\ Patients and controls}

This study included a group of 92 patients of Portuguese origin belonging to 60 apparently unrelated families; the patients were diagnosed with different types of VWD between 2007 and 2014. Sixty probands and 32 affected relatives (parents and siblings) were distributed as follows: 56 patients (31 families) regularly attended the Department of Haematology at Centro Hospitalar Universitário de Coimbra for clinical and laboratorial follow-up. The remaining 36 patients (29 families) were referred from external centres in different regions of Portugal: Lisbon (three central hospitals, one paediatric) and Faro (one central hospital). These hospitals all diagnose and treat patients with VWD and had sent samples from patients with different types of VWD to our centre for confirmation of their results, for multimer pattern analysis and for molecular study. The patients included in this study were from the central and southern regions of Portugal.

The clinical characteristics of patients were recorded through a validated questionnaire focusing on personal and family history of significant mucocutaneous haemorrhage using bleeding scores (BSs) calculated with the bleeding assessment tools (BAT) recommended by the International Society on Thrombosis and Haemostasis and Standardization Committee on von Willebrand Factor guidelines (12). In accordance with the Declaration of Helsinki, informed consent was obtained from all probands and their family members.

The 92 individuals in this study included both adults and children with a median age of 28 years (1-76) and a sex distribution of $58 \mathrm{~F}: 34 \mathrm{M}$. The adult probands $(\mathrm{n}=42)$ had a median age of 41 years with a range of 19-76 years, and the child probands (n $=18$ ) had a median age of 8 years with a range of $1-15$ years.

Forty-two healthy volunteers acted as a control group for measurements of the PFA-100 closure time (PFA:ADP; PFA:Epi), FVIII coagulant activity (FVIII:C), VWF antigen level (VWF:Ag), ristocetin cofactor activity (VWF:RCo) and/or VWF collagen binding (VWF:CB) ( Table 1). The control group included unrelated individuals $(32 \mathrm{~F}: 10 \mathrm{M})$ without an individual or family history of mucocutaneous haemorrhage, with a mean age of $33.5 \pm$ $11.12 \mathrm{yrs}$.

\section{Samples and sample processing}

Functional and immunological studies were performed on blood collected into vacuum tubes containing $3.2 \%$ sodium citrate and centrifuged within 15 minutes ( $\mathrm{min}$ ) at room temperature for 20 $\mathrm{min}$ at $2500 \mathrm{~g}$. The obtained platelet-poor plasma was then separated into aliquots and kept frozen at $-80^{\circ} \mathrm{C}$ until use. The separated plasma was later subjected to coagulation and multimer assays. Genomic DNA was extracted from EDTA whole blood by automatic isolation on an $\mathrm{iPrep}^{\mathrm{TM}}$ instrument using a gDNA Blood Kit (Invitrogen, Carlsbad, USA). The DNA concentration was adjusted to a range of $25-50 \mathrm{ng} / \mu \mathrm{l}$.

\section{Algorithm for phenotypic analysis - coagulation and multimer assays}

VWF analysis was performed according to an algorithm based on established recommendations (6): i) screening tests - platelet function analysis via the PFA-100 closure time (Siemens Healthcare, Marburg, Germany) in addition to measurements of the FVIII:C level, VWF:Ag level, VWF-platelet GPIb binding activity (Innovance) and VWF:RCo that were performed on a BCS XP coagulation analyser according to the manufacturer's instructions (Siemens Healthcare, Marburg, Germany); ii) specific tests - to determine disease subtype, ristocetin-induced platelet aggregation (RIPA) in platelet-rich plasma with final ristocetin concentrations of $0.5 \mathrm{mg} / \mathrm{ml}$ and $1.2 \mathrm{mg} / \mathrm{ml}$ was performed using an in-house aggregometry assay. VWF:CB was measured (except in type 3 and $2 \mathrm{~N}$ VWD) using type III collagen (Technozym, Technoclone, Vienna, Austria), and the capacity of VWF to bind exogenous 
Table 1: Demographic and phenotypic data of 60 probands at diagnosis.

\begin{tabular}{|c|c|c|c|c|c|}
\hline & $\begin{array}{l}\text { Type } 1 \\
(n=7)\end{array}$ & $\begin{array}{l}\text { Type } 3 \\
(n=15)\end{array}$ & $\begin{array}{l}\text { Type } 2 N \\
(n=12)\end{array}$ & $\begin{array}{l}\text { Type } 2 A, 2 B, 2 M \\
(n=25)\end{array}$ & P-value \\
\hline Male/Female & $4 / 3$ & $1 / 14$ & $5 / 7$ & $8 / 17$ & 0.0384 \\
\hline Median age, years (range) & $17(1-34)$ & $15(1-55)$ & $41(13-69)$ & $36(2-76)$ & NS \\
\hline Median bleeding score (range) & $14(5-18)$ & $18(10-23)$ & $12.5(2-21)$ & $11(1-24)$ & NS \\
\hline $\begin{array}{l}\text { PFA (COL/ADP), s } \\
\text { PFA (COL/Epi), s }\end{array}$ & $\begin{array}{l}>300 \\
>300\end{array}$ & $\begin{array}{l}>300 \\
>300\end{array}$ & $\begin{array}{l}121(99-188) \\
96(82-125)\end{array}$ & $\begin{array}{l}>300 \\
>300\end{array}$ & NS \\
\hline FVIII:C* (\%) (range) & $26(11-33)$ & $2.1(<0.25-6)$ & $27.5(7-38)$ & $37.5(15-100)$ & $<0.0001$ \\
\hline VWF:Ag* (\%) (range) & $9.9(3-16)$ & $<4$ & $68.0(45-120)$ & $30.0(14-85)$ & $<0.0001$ \\
\hline VWF:RCo* (\%) (range) & $7.2(2-15)$ & $<4$ & $64.0(47-100)$ & $11.0(<4-32)$ & $<0.0001$ \\
\hline VWF:RCo/VWF:Ag* (range) & $0.8(0.67-1.00)$ & - & $1.0(0.83-1.11)$ & $0.4(0.14-0.73)$ & $<0.0001$ \\
\hline VWF:CB* (\%) (range) & $4.5(2-16)$ & - & - & $13.0(<4-53)$ & $<0.0001$ \\
\hline
\end{tabular}

Normal range: PFA (COL/ADP), 93-161 s; PFA (COL/Epi), 67-99 s; FVIII:C, 49-149\%; VWF:Ag, 50-160\%; VWF:RCo, 48-173\% and VWF:CB, 60-130\%. PFA-platelet function analysis; s - seconds; *Median and range; Chi-squared testing for categorical variables; One-way ANOVA followed by Bartlett's test for all linear variables; NS, no significance.

FVIII (VWF:FVIIIB) (Stago, Asnieres, France) was evaluated using ELISA. The multimer pattern was evaluated by SDS-agarose gel electrophoresis (low-resolution gel electrophoresis using $0.9 \%$ low-gelling temperature agarose or medium resolution using $1.6 \%$ agarose), followed by Western blotting and detection with rabbit anti-human VWF antibody (Dako, Glostrup, Denmark) using the alkaline phosphatase staining method (13).

The potential probands with VWF deficiency were diagnosed by evaluating the following criteria: presence of reduced $(<$ $30 \%)$ VWF:RCo levels; a discrepant VWF:RCo/VWF:Ag ratio of $<0.7$ that suggested type 2 VWD; a discrepant FVIII:C/VWF:Ag ratio of $<0.5$ that suggested type $2 \mathrm{~N}$ VWD; the absence of VWF (or presence of only trace amounts) and a FVIII:C below $5 \%$ that distinguished type 3 VWD from severe type 1 VWD (1).

The rate of VWF mutations found in type 1 VWD decreases when the VWF level is $>30 \%$ (1). To avoid high costs, in our approach, patient samples with borderline VWF levels were not pursued for molecular analysis and Sanger sequencing.

\section{Alloantibodies to VWF}

To test alloantibodies to VWF in type 3 patients, an in-house ELISA-based assay was used as previously described (14).

\section{Strategy for VWF mutation analysis}

The molecular analysis in this study used two different direct sequencing methodologies:

i) Sanger direct sequencing: For the seven years of this study, the following sequences were determined using an approach based on VWD subtypes: type 1 severe and type 3 VWD - all coding VWF; types $2 \mathrm{~B}$ and $2 \mathrm{M}$ VWD - only exon 28 ; type $2 \mathrm{~A}$ - first exon 28 and then exons 11-16, 24-26 and 51-52 if there was no mutation in exon 28; and type $2 \mathrm{~N}$ - exons $18-25$. To confirm differential diagnosis between VWD 2B and platelet type VWD (PT-VWD), exon 2 of GP1BA.

ii) NGS: The coding VWF [regions of interest (ROIs)] were analysed in all samples (DNA samples were sent to Unitat de Diagnòstic i Teràpia Molecular, Banc de Sang i Teixits (BST, Barcelona, Spain)).

\section{Sequencing of VWF using NGS and identification of genetic variants}

A recently described automated method for the Access Array ${ }^{\mathrm{m}}$ platform (Fluidigm, South San Francisco, CA, USA) derived from a previous NGS protocol $(10,15)$ was used for VWF amplification. Briefly, the resulting products from all patients included in this study were pooled and simultaneously sequenced in a MiSeq Desktop Sequencer (Illumina, San Diego, CA, USA) run. Barcoded sequences were de-multiplexed and individually analysed. The ROIs included in our NGS design were approximately $1300 \mathrm{bp}$ of the VWF promoter region, exons 1-52 and the intronic flanking regions (at least $20 \mathrm{bp}$ ). The depth of coverage and primer design is described in detail in the Suppl. Material (available online at www.thrombosis-online.com). The NGS pipeline output, in paired sequence files (FASTQ format), was used as an input for the analysis, which began with CLC Genomic Workbench software (Qiagen, Hilden, Germany) and then proceeded to VariantStudio (Illumina). The optimal analysis parameters (coverage, minor allele counts, percent of variant alleles, etc.) were adjusted to obtain the optimal performance for variant detection (see Suppl. Material, available online at www.thrombosis-online.com). This workflow allows the alignment of the resulting sequences against the human genome sequence (hg19) and concurrent in silico analysis, permitting the identification of potential pathogenic variants, discriminating pseudogene sequences and filtering the known 
polymorphisms described to date in the dbSNP (16) and 1000 Genomes databases (17).

For confirmation of the variants identified by NGS, the corresponding region was amplified using PCR and sequenced by Sanger direct sequencing, as described in the Suppl. Material (available online at www.thrombosis-online.com).

\section{Multiplex ligation-dependent probe amplification (MLPA)}

DNA samples from patients lacking identified pathogenic variants using PCR/direct sequencing, were screened using SALSA MLPA P011 and P012 VWF kits (version B2; MRC-Holland) to detect deletions/duplications in VWF. Fragment size analysis was performed using an ABI 3130 Genetic Analyzer (Applied Biosystems, Foster City, CA, USA). Data normalisation was performed using four healthy controls and Coffalyser. Net software (MRC-Holland) was used for analysis using the fragment analysis files (.fsa) obtained from the Applied Biosystems Genetic Analyzer as input.

\section{Mapping of the VWF exon 31 deletion breakpoints}

The exact locations of the deletion breakpoints in one type 1 VWD proband in whom the mutation was initially identified by MLPA were determined by primer walking followed by Sanger sequencing. The genomic region from the end of exon 30 to intron 31 was amplified using five primer sets. The following primer pair sequences were used in the specific assay for the detection of the deletion: forward primer 5'-GCTGCAGTATGGAAGCATCA-3' and reverse primer 5'-GAGTTGAGGTGAGGCTGGAG-3'.

\section{In silico analysis}

The impact of missense changes was further analysed according to the recommended criteria that included evolutionary conservation of an amino acid or nucleotide, location and context within the protein sequence and the biochemical consequence of the amino acid substitutions $(18,19)$. This analysis was assessed using five different in silico algorithms: PROVEAN, SIFT, PolyPhen-2, MutationAssessor and MutationTaster (Suppl. References, available online at www.thrombosis-online.com) (last accessed September 21, 2015). Missense variants were considered deleterious if at least three of the five prediction programs suggested a pathogenic effect.

Potential disease-causing variants due to aberrant splicing were evaluated using a set of five different in silico algorithms: Human Splicing Finder (HSF), MaxEntScan, NNSPLICE, NetGene2 and Splice View (Suppl. References, available online at www.thrombo sis-online.com) (last accessed September 21, 2015). This approach improves the accuracy of the splicing analysis of intronic, deep intronic and exonic variants that may directly disrupt constitutive splice sites or indirectly create cryptic splice sites $(20,21)$. MutPred Splice was used to confirm probable exonic splicing variants (22). A local splice site effect was considered potentially deleterious when at least three (intronic variants) or four (exonic variants) of these predictions for the variant vicinity were significant.

\section{Genetic databases}

As recommended by the guidelines $(18,21,23)$, we defined those variants that are not reported in the international databases (populationand disease-specific) as well as in published literature as 'novel.' Therefore, in addition to using the polymorphism databases (dbSNP and 1000 Genomes databases) mentioned above, we checked variants for inclusion in the Exome Aggregation Consortium (ExAC) (24), Exome Variant Server (EVS) (25), Human Gene Mutation Database (26) and VWF international mutation databases (27-29).

\section{Assessment of the pathogenicity of variants}

The following criteria were used to evaluate the pathogenicity of the variants: 1) whether the variant was a stop/frameshift variant, which was considered to most likely be disease causing, 2) cosegregation in the family, 3 ) whether the variation had been previously identified in international databases, 4) in silico evaluation and 5) presence of the second mutant allele in the case of autosomal recessive inheritance.

Variants were classified as pathogenic, likely pathogenic, uncertain significance, likely benign or benign based on the available evidence, according to the practice guidelines for the evaluation of pathogenicity recently published by the American College of Medical Genetics and Genomics and the Association for Molecular Pathology (18).

The amino acid numbering and nomenclature used is according to the international recommendations for the description of sequence variants of the Human Genome Variation Society (http://www.HGVS.org) and applied to genetic variants in haemostasis (30).

\section{Statistical analysis}

Differences between groups were analysed by Chi-squared testing for categorical variables and one-way ANOVA followed by Bartlett's test for all linear variables. Tests were assumed significant whenever the two-tailed $\mathrm{p}$-value was $<0.05$. These statistical analyses were performed using GraphPad Prism 6.0 for Windows (GraphPad Software, La Jolla, CA, USA; www.graphpad.com).

\section{Results \\ Laboratory haemostasis findings}

The present study included a cohort of 60 probands from different regions of Portugal with severe/intermediate types of VWD. According to the study algorithm, in the first approach, a standardised BS and VWF:Ag, VWF:RCo and FVIII:C levels were taken into account to diagnose these patients. BS showed an overall inverse correlation with the plasma levels of VWF:Ag ( $\mathrm{p}=0.0022$, Spearman's rho $=0.3822)$, VWF:RCo $(p=0.0013$, Spearman's rho $=0.3991)$ and FVIII:C $(p=0.0020$, Spearman's rho $=0.3829)$.

Forty-eight probands presented with low levels of VWF:RCo $(9.15 \pm 9.19 \%)$, and 12 presented with a reduced FVIII:C/VWF:Ag 
Table 2: Phenotypic and molecular data of seven probands with severe type 1 VWD.

ratio $(0.36 \pm 0.15)$; these features prompted a detailed phenotypic study and mutational analysis.

The phenotypic characteristics of the probands were mainly distributed as follows: severe type $1 \mathrm{VWD}(\mathrm{n}=7)$, type $3 \mathrm{VWD}(\mathrm{n}$ $=15)$ and type 2 VWD $(n=38)$. Specific tests indicated the subdivision of type 2 VWD as follows: type $2 A(n=9)$, type $2 B(n=$ $6)$, type $2 \mathrm{M}(\mathrm{n}=11)$ and type $2 \mathrm{~N}(\mathrm{n}=12)(-$ Table 1$)$. Familial studies $(\mathrm{n}=32)$ included 25 affected relatives, three type 3 carriers and four type $2 \mathrm{~N}$ carriers (data not shown).

The NGS method confirmed all variants detected by the Sanger method (100\% sensitivity) and detected 26 additional variants: six missense, three synonymous and 17 intronic [15 of $17(88 \%)$ were deep intronic]. In total, 62 different variants, whose frequency in the different populations studied in 1000 Genomes was below $1 \%$, are summarised in Suppl. Table 1 (available online at www.throm bosis-online.com): 30 missense variants $(48.5 \%)$, three nonsense (4.8\%), two small deletions (3.2\%), one large deletion (1.6\%), one duplication (1.6\%), five synonymous $(8 \%)$ and 20 intronic variants $(32.3 \%)$. The frequencies of those variants were also checked in the ExAC and EVS_EA population databases, which revealed differing values for some variants (Suppl. Table 1, available online at www.thrombosis-online.com).

In total, 27 variants had never been reported in the population databases and international VWF databases (Suppl. Table 1, available online at www.thrombosis-online.com).

\section{Prediction of pathogenic variants}

Twenty-six out of 31 missense variants were predicted to be deleterious (Suppl. Table 2, available online at www.thrombosis-on line.com). Fifteen of 56 variants (missense, synonymous and intronic) were detected using some in silico tools with probable impact on splicing; however, only six reached the required score $(\geq 3$ for intronic and $\geq 4$ for exonic mutations) and were considered potential splice site variants (Suppl. Table 3, available online at www.thrombosis-online.com). According to the practice guidelines (18), these in silico analyses combined with other evidence data (population, functional and segregation) permitted the following classification of the variants: pathogenic $(n=29 ; 46.9 \%)$, likely pathogenic $(\mathrm{n}=4 ; 6.4 \%)$, uncertain significance $(\mathrm{n}=4$; $6.4 \%)$, likely benign $(\mathrm{n}=18 ; 29 \%)$ and benign $(\mathrm{n}=7 ; 11.3 \%)$ (Suppl. Table 1, available online at www.thrombosis-online.com). Accordingly, for the classification of these variants, we used the term 'mutation' only to refer to pathogenic variants.

Variants were identified in all probands of this cohort $(100 \%)$, and $29(48.3 \%)$ had two or more variants along VWF.

\section{Potential functional impact of novel mutations}

Eleven of the 27 novel variants (41\%) were pathogenic: five missense changes (c.440A $>\mathrm{G}$, p.Gln147Arg; c.2637C >A,

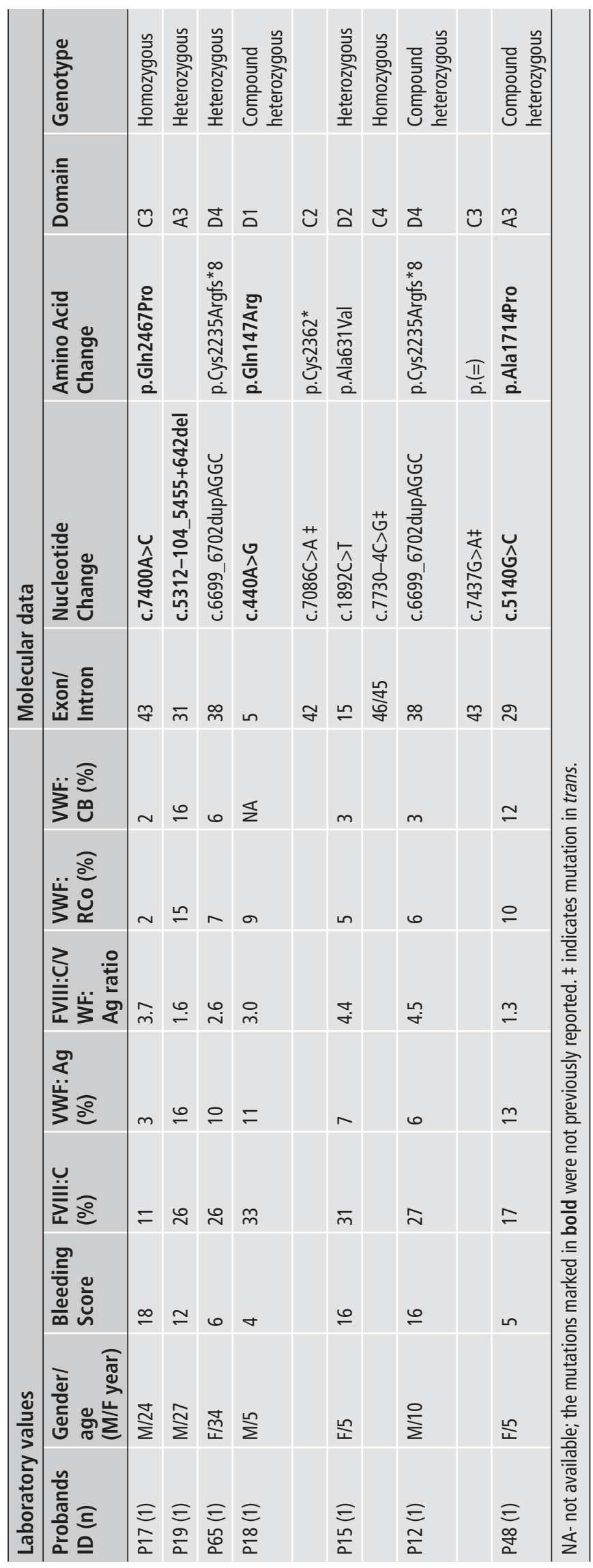

Thrombosis and Haemostasis 116.1/2016 
p.Asp879Glu; c.4117G>T, p.Asp1373Tyr; c.5140G $>$ C, p.Ala1714Pro; and c.7400A>C, p.Gln2467Pro); two nonsense mutations (c.4666C > T, p.Gln1556*; c.7086C>A, p.Cys2362*); two small deletions (c.100delT, p.Arg34Aspfs ${ }^{\star} 49$; c.5414_5415del, p.Val1805Glyfs ${ }^{\star} 8$ ); one large deletion [c.5312-104-_5455+642del (890 bp)] and one potential splice site mutation at the consensus 5'-GT donor splice site (c.1533+1G>A) (Suppl. Table 1, available online at www.thrombosis-online.com). The remaining 16 variants accounted for two missense changes that were signalled as benign variants (c.3590C $>$ A, p.Pro1197Gln; c.3686T $>$ C; p.Val1229Ala) and 14 deep intronic variants that were considered with uncertain significance (2) and likely benign (12) (Suppl. Table 1, available online at www.thrombosis-online.com).

\section{Phenotype-genotype correlations}

The probands' phenotypic and molecular data were separated according to the VWD type, and only the variants with predicted pa- thogenicity (mutations) are summarised in $>$ Tables 2-6. For four probands, the phenotype-based classification was changed after mutation analysis.

\section{Type 1 VWD}

Seven probands had a severe type 1 VWD profile with very low VWF:Ag and VWF:RCo levels but with moderately reduced FVIII:C levels ( Table 2). In fact, five of these seven patients had an increased FVIII:C/VWF:Ag ratio with a median value of 3.7 (range 2.6-4.5). Thirteen variants were distributed along several regions of $V W F$ with alterations in domains D1, D2, A3, D4, C2, C3 and C4 ( Figure 1). As shown in $>$ Table 2, 10 mutations (five not previously described) were associated with this phenotype. All probands except three had compound heterozygous mutations: one had a novel homozygous missense mutation, p.Gln2467Pro, located in the C3 domain, another had a novel heterozygous large deletion of exon 31 located in the A3 domain, and the third had a

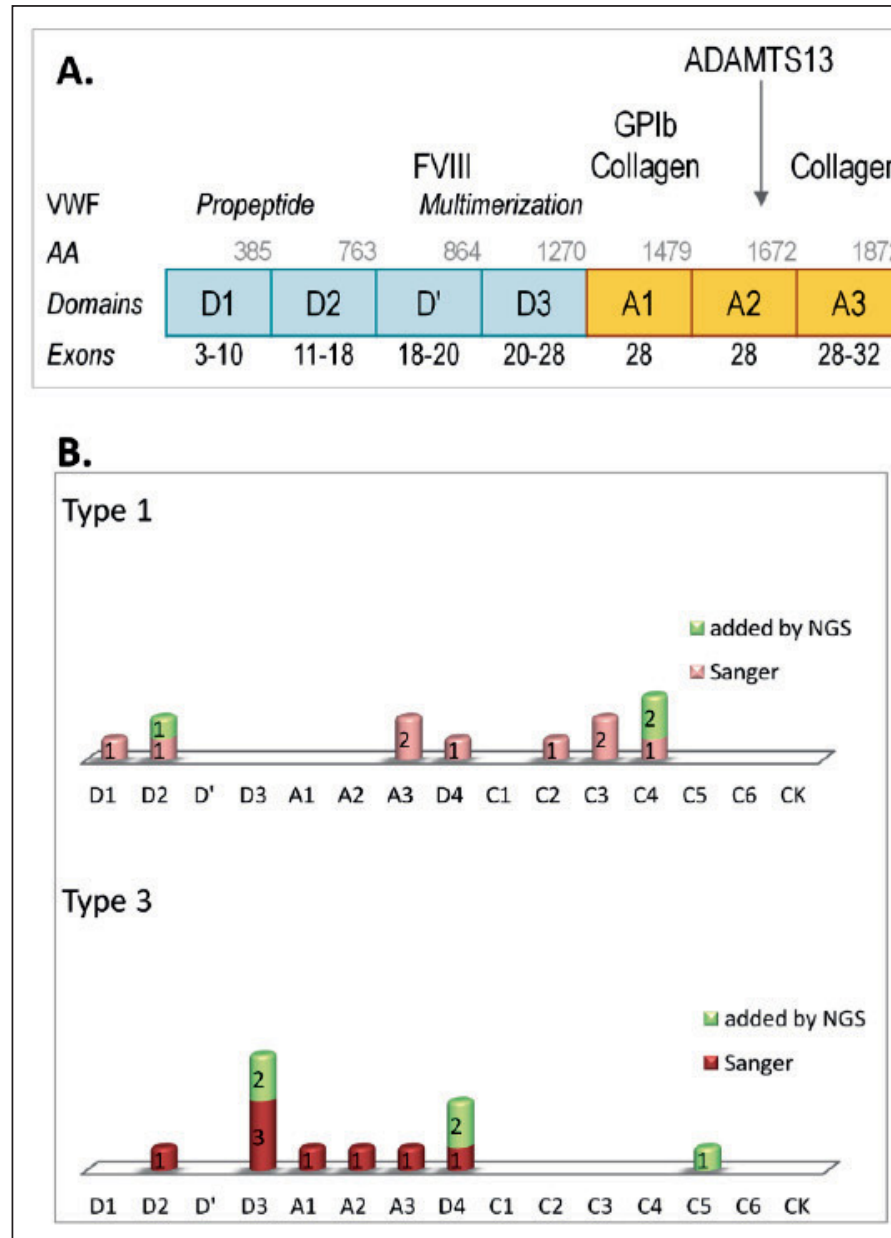

Figure 1: Distribution of 62 different VWF mutations identified in 60 probands with VWD. A) The scheme represents VWF and the corresponding domains of the VWF protein $(55,56)$. B) The graphics show the mutation distribution associated with the VWF domains for severe type 1, type 3, type $2 \mathrm{~N}$ and type 2A, 2B, 2M VWD. Each graphic discriminates between the mutations previously identified by Sanger sequencing and the mutations added
Type $2 \mathrm{~N}$

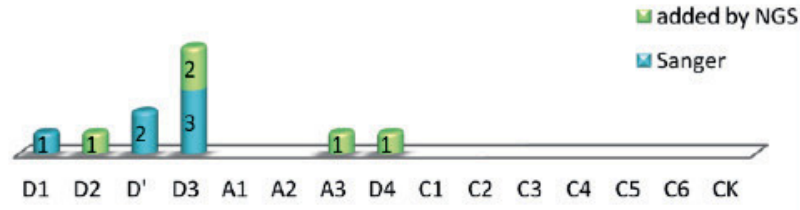

Type 2A, 2B, 2M

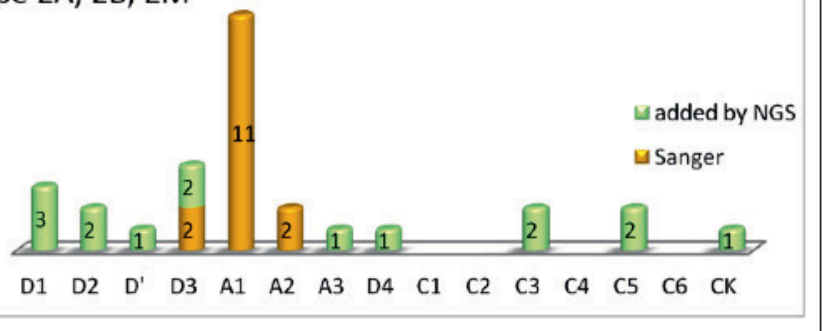

by NGS. No evident cluster of alterations was observed for type 1 and type 3 VWD, and the spreading along VWF was accentuated. For type $2 \mathrm{~N}$ VWD, a mutation cluster in the region coding for $D^{\prime}-D 3$ was preserved. For type $2 A$, $2 B$ and $2 M V W D$, mutation spreading was evident, but a mutation cluster emerged in the region coding for the $A 1$ domain. 
duplication mutation c.6699_6702dupAGGC, p.Cys2235Argfs ${ }^{\star}$. MLPA analysis detected the large deletion, and PCR followed by direct DNA sequencing mapped the intronic breakpoints c.5312-104_5455+642del, indicating that the deletion was 890 bp in size (Suppl. Figure 1, available online at www.thrombosis-on line.com).

The remaining four probands showed compound heterozygosity in trans for missense and null mutations (nonsense and splice site). Two probands had a recessively inherited missense mutation p.[Gln147Arg];[Cys2362*] and [p.Ala631Val];[c.7730-4C>G] located in the VWF propeptide (VWFpp) region (D1-D2 domain). Both had a typical multimeric pattern with a heavy predominance of VWF dimers (IIC). The proband P12 had a duplication mutation c.6699_6702dupAGGC, p.Cys2235Argfs ${ }^{*} 8$ and the synonymous mutation c.7437G $>\mathrm{A}$ in the splice region, resulting in severely decreased VWF:Ag levels. The same duplication mutation was detected in the homozygous state in type 3 VWD patients ( Table 3). Finally, the proband P48 had a compound heterozygosity in trans for the novel missense mutation p.Ala1714Pro in the $\mathrm{A} 3$ domain and the potential splice site variant $\mathrm{c} .5170+10 \mathrm{C}>\mathrm{T}$. This missense mutation in the proband's mother was inherited as a dominant trait with a type $2 \mathrm{M}$ VWD phenotype, normal multimeric pattern and low VWF:CB/VWF:Ag ratio (0.54), thus showing a pleiotropic effect.

\section{Type 3 VWD}

The severity of 15 probands with type 3 VWD was evidenced by the low median age ( 15 years) and higher BSs, which were consistent with the low levels of FVIII:C (median $2 \%$, range $<0.25-6$ ), VWF:Ag $(<4 \%)$ and VWF:RCo $(<4 \%)$ and the absent VWF multimeric pattern ( Table 1 and $>$ Table 3$)$. Mutations were identified in 14 probands, 13 of whom were homozygous for only one mutation (93\%). In total, 13 different variants and six mutations were scattered throughout VWF ( Figure 1).

Three novel mutations were identified in our cohort of type 3 VWD patients: the nonsense mutation c.4666C $>\mathrm{T}$ (p.Gln1556*), the small deletion c.5414_5415delTG (p.Val1805Glyfs ${ }^{\star} 8$ ) and the splice site mutation c. $1533+1 \mathrm{G}>\mathrm{A}$.

The majority of the probands $(13 / 15 ; 87 \%)$ were homozygous for a null mutation that comprised the nonsense mutations c.3931C > T (p.Gln1311*) and c.4666C > T (p.Gln1556*), the small deletion c.5414_5415delTG (p.Val1805Glyfs ${ }^{\star} 8$ ) and the duplication c.6699_6702dupAGGC (p.Cys2235Argfs ${ }^{\star} 8$ ). One proband was compound heterozygous for [p.Tyr1146Cys];[c.1533+1G>A]; a study of the parents allowed the tracing of the mutation inheritance in each allele: the father was heterozygous for p.Tyr1146Cys, showing a mild type $1 \mathrm{VWD}$, whereas the mother was heterozygous for c. $1533+1 \mathrm{G}>\mathrm{A}$ but was asymptomatic.

The remaining type 3 VWD proband and her brother were homozygous for a dinucleotide change c.3485_3486delinsTG that results in the variant p.Pro1162Leu, which affects the D3 domain; however, the real contribution of this variant to the severe quantitative phenotype must still be clarified. Thus, causative mutations for type 3 VWD were found in 14 out of 15 probands (93\%).
Table 3: Phenotypic and molecular data of 14 probands with type 3 VWD.

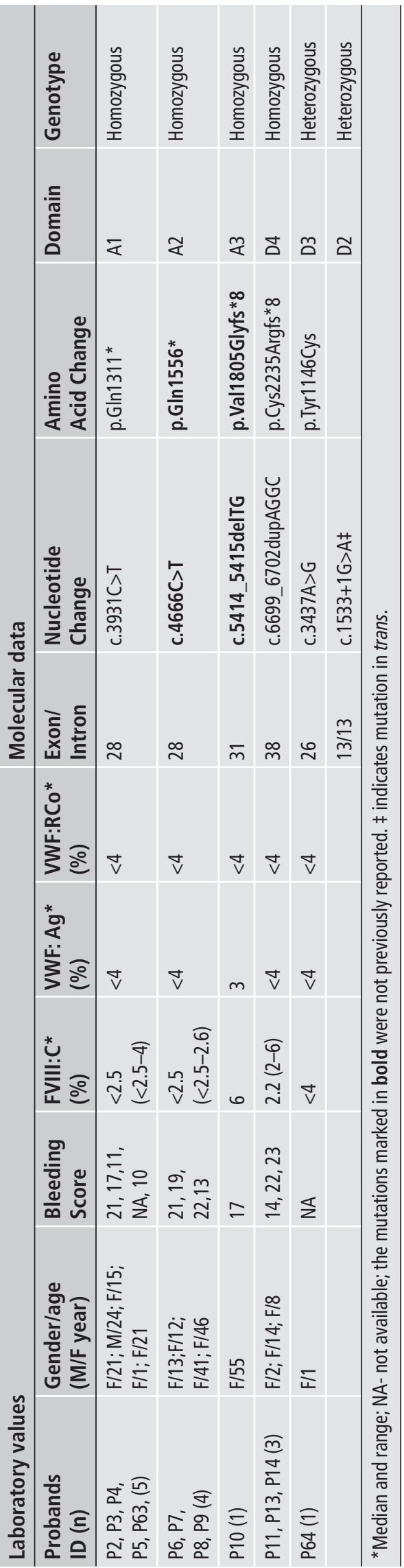




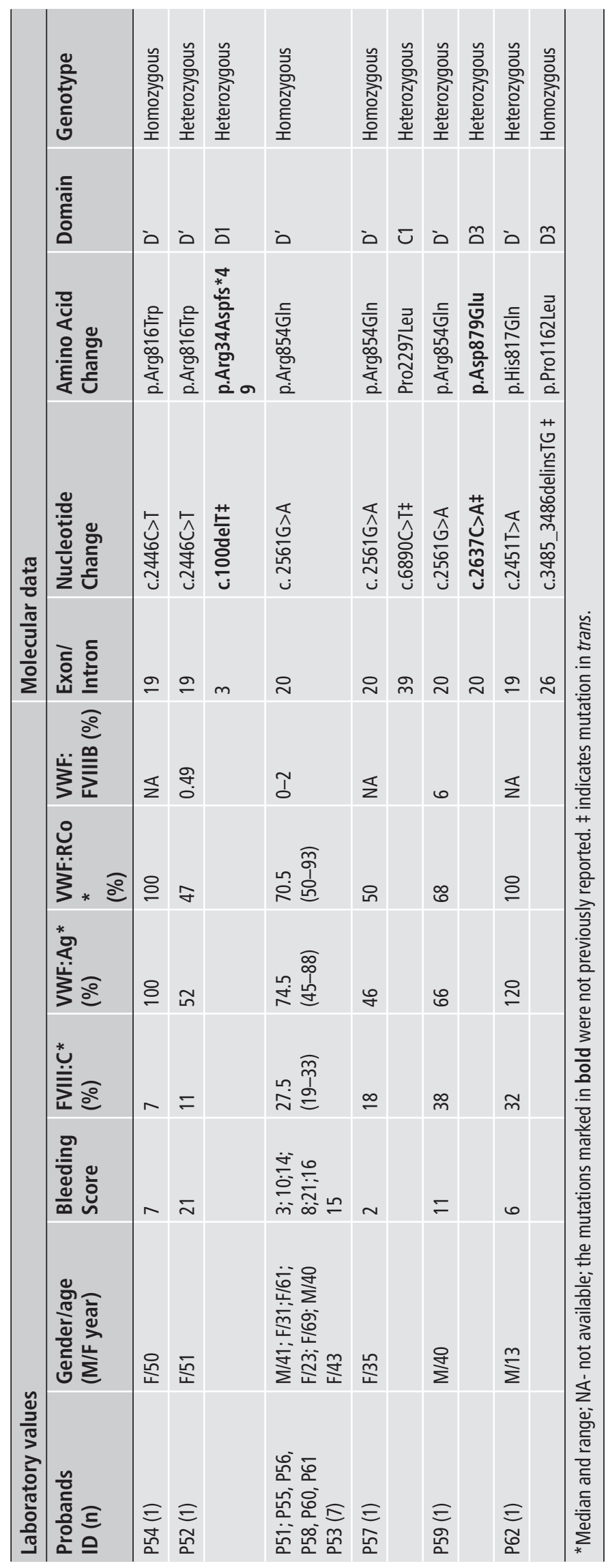

Table 4: Phenotypic and molecular data of 12 probands with type $2 \mathrm{~N}$ VWD.

Among those patients who were homozygous for the c.3931C>T (p.Gln1311*) mutation, one proband (P2) showed an ineffective response after replacement therapy, and the presence of an inhibitory alloantibody against VWF was confirmed by an anti-VWF ELISA assay. On the basis of these data, the patient received FVIII bypass therapy according to their clinical situation.

\section{Type 2N VWD}

All the 12 type $2 \mathrm{~N}$ VWD probands had reduced FVIII:C levels with median levels of $27.5 \%$ (range 7-38), normal or near normal median VWF:Ag levels of approximately 68\% (range 45-120), normal VWF:RCo levels of $64 \%$ (range 47-100) and a normal VWF multimeric pattern (Table 1). The reduced FVIII:C/VWF:Ag ratio $(0.4 ; 0.07-0.6)$ was suggestive of type $2 \mathrm{~N}$ VWD, which was confirmed by reduced VWF:FVIII binding and/ or molecular studies.

In total, 11 different variants and seven mutations were found in the $V W F$ regions affecting the domains D1, D, D3, A3 and D4. Despite this distribution, five missense mutations were clustered in exons 19 and 26 of VWF, which encode the FVIII-binding D'-D3 domains ( Figure 1). The frequently described missense mutations c.2561G >A (p.Arg854Gln) and c.2446C >T (p.Arg816Trp) repetitively occurred in our cohort, being the most recurrent mutations ( $75 \%$ of cases) ( $>$ Table 4$)$.

The missense mutation p.Arg816Trp was found in the two probands who had the lowest FVIII:C levels ( Table 4), one in a homozygous state and the other compound heterozygous with a null allele p. [Arg816Trp];[Arg34Aspfs $\left.{ }^{\star} 49\right](2 \mathrm{~N} / 3)$. Eight out of nine individuals were homozygous for p.Arg854Gln, and one was compound heterozygous for a novel mutation, c.2637C $>\mathrm{A}$ (p.Asp879Glu).The FVIII:C/VWF:Ag ratio was not significantly different in homozygotes or heterozygotes for the p.Arg854Gln mutation. In a 13-year-old African male (P62) who was previously diagnosed with mild haemophilia A (based only on functional studies), heterozygosity for the missense variant c.2451T >A (p.His817Arg) and homozygosity for the variant c.3485_3486delinsTG (p.Pro1162Leu) were detected in the D' and D3 domains, respectively. The two variants, p.Pro1162Leu and p.His817Arg, located in the VWF region, which affects the FVIII binding capacity, could both contribute to the type $2 \mathrm{~N}$ phenotype.

In this group of $2 \mathrm{~N}$ VWD patients, two probands (P61 and P62) had previously been misclassified as having mild haemophilia A.

\section{Type 2B VWD}

The six probands classified as $2 \mathrm{~B} V W D$ had a very low median VWF:RCo level of 11\% (range 10-24) and a VWF:RCo/VWF:Ag 
ratio of 0.4 (0.35-0.50); the VWF multimeric patterns showed partial or complete loss of the HMW multimers ( Table 5). Among the six probands, four had thrombocytopenia $(15,50,79$ and $130 \times$ $10^{3}$ platelets/ $\left.\mu \mathrm{l}\right)$. Because the samples from three probands were provided by other centres, it was only possible to perform RIPA in the other three patients who showed an enhanced platelet aggregation in response to $0.5 \mathrm{mg} / \mathrm{ml}$ of ristocetin.

The frequently described mutations associated with VWD 2B, p.Arg1306Trp and p.Val1316Met, in the region coding for the A1 VWF domain, were found in five patients ( $>$ Table 5 and $>$ Figure 1 ). One proband (P25) was referred to us with a suspected diagnosis of Bernard-Soulier syndrome because of the severe thrombocytopenia $\left(15 \times 10^{3}\right.$ platelets $\left./ \mu \mathrm{l}\right)$ and the giant platelets observed in a peripheral blood smear. However, the coagulation multimer assays and molecular studies allowed a reclassification as type $2 \mathrm{~B}$ VWD with a compound heterozygous in cis for mutations p.[Arg1306Trp;Arg854Gln]. The proband P50 was compound heterozygous in trans for p.Val1316Met with two other variants, the novel benign missense variant p.Pro1197Gln and c.5170+10C $>\mathrm{T}$.

Finally, in proband P66, despite the VWF levels being consistent with type 2B VWD, no mutation was found in exon $28 \mathrm{VWF}$. The phenotype data included low VWF:RCo levels (47\%), a VWF:RCo/VWF:Ag ratio of 0.4 , partial loss of the HMW multimers and RIPA at $0.25 \mathrm{mg} / \mathrm{ml}$. A differential diagnosis of probable platelet-type VWD (PT-VWD) was performed using simple RIPA mixing studies (control platelets in patient plasma), which revealed the absence of aggregation with $0.5 \mathrm{mg} / \mathrm{ml}$ of ristocetin. Therefore, a platelet-type phenocopy (pseudo-VWD) was considered. The sequencing of GP1BA revealed the presence of a previously described missense mutation, c.751G > T, p.Asp251Tyr in the heterozygous state in exon 2 , confirming the reclassification of this patient as PT-VWD (Suppl. Figure 2, available online at www.thrombosis-online.com).

\section{Type 2A and 2M VWD}

The VWF:RCo and VWF:Ag levels corresponded to discrepant VWF:RCo/VWF:Ag ratios $(0.39 \pm 0.22)$ in these two groups of patients. The VWF:CB levels were similar to the VWF:RCo levels for type 2A VWD and to the VWF:Ag levels for 2M VWD. The VWF multimeric patterns in the type 2A VWD group showed the loss of the HMW multimers, and the pattern for the 2M VWD group showed the presence of all-size multimers ( Table 6). Probands with type $2 \mathrm{~A}$ VWD had a significantly higher BS (median $=15)$ than those with type $2 \mathrm{M}$ VWD (median $=8.5)(\mathrm{p}=0.020$, Mann-Whitney U test).

In 20 probands, 10 different mutations were detected in exon 28, which encodes the VWF A1 and A2 domains (>Figure 1). Among these five mutations (p.Arg1374Cys p.Arg1374His, p.Arg1399Cys, p.Ser1506Leu and p.Ile1628Thr) that occurred repetitively, p.Ile1628Thr was the most frequent mutation in $2 \mathrm{~A}$ VWD (40\% of cases) and p.Arg1374His was the most frequent in $2 \mathrm{M} \mathrm{VWD}$ (37\% of cases) ( Table 6 ).

Of the nine probands with a $2 \mathrm{~A}$ VWD phenotype, only one (P20) was heterozygous for the missense mutation in exon 26 (do- main D3), c.3388T>C p.Cys1130Arg, with low levels of VWF:RCo (16\%), a discrepant VWF:RCo/VWF:Ag ratio of $<0.7$ and a characteristic 2A (IIE) VWF multimeric pattern (absence of large multimers and no triplet structure of individual bands), which is indicative of a multimerisation defect ( Table 6, Suppl. Figure 3, available online at www.thrombosis-online.com).

The probands P23 and P24 were heterozygous for two mutations affecting the same position and located in the first amino acid of the A1 loop domain: c.3814T $>$ G, p.Cys1272Gly and c.3815G $>$ T, p.Cys1272Phe. Interestingly, the mutation p.Cys1272Phe showed compound heterozygosity with a potential splice site variant (c.7288-68G $>$ A) that could strongly activate a cryptic donor (Suppl. Tables 1 and 3, available online at www. thrombosis-online.com). Despite this mutation location being less common in type $2 \mathrm{~A} \mathrm{VWD}$, the laboratory phenotype was consistent in these two probands: a marked decrease in VWF:RCo levels (5\%-7\%), a VWF:RCo/VWF:Ag ratio of < 0.18 , a multimeric pattern showing a loss of high- and intermediate-molecular weight multimers (Suppl. Figure 3, available online at www.thrombosis-online.com) and RIPA at $1.25 \mathrm{mg} / \mathrm{ml}$ was absent.

The remaining six probands were heterozygous for two frequently described missense mutations clustered in the A2 domain: c.4517C>T, p.Ser1506Leu $(\mathrm{n}=2)$ and c.4883T $>$ C, p.Ile1628Thr (n =4). The two patients heterozygous for the p.Ser1506Leu mutation, described as a VWD 2A group I defect with intracellular proteolysis of large VWF multimers, showed a similar multimeric pattern (Suppl. Figure 3, available online at www.thrombosis-on line.com). These probands had moderate/severe type 2A VWD with low levels of VWF:Ag (median 17.5\%) and VWF:RCo (median $11 \%$ ), a low VWF:RCo/VWF:Ag ratio (median 0.64 ) and no RIPA at $1.25 \mathrm{mg} / \mathrm{ml}$. The remaining four probands were heterozygous for p.Ile1628Thr, which has been described as a VWD 2A group II defect characterised by hypersensitivity to ADAMTS13 with increased proteolysis in plasma (5). They had mild/moderate type 2A VWD with low levels of VWF:Ag (median 54.8\%) and VWF:RCo (median 13.2\%), a low VWF:RCo/VWF:Ag ratio (median 0.2) and normal RIPA at $1.25 \mathrm{mg} / \mathrm{ml}$. These four patients showed a typical proteolytic pattern with a lack of large VWF multimers and the presence of a pronounced triplet structure, which is characteristic of group 2A/II.

Of the 11 probands with the type $2 \mathrm{M}$ VWD phenotype, eight $(72 \%)$ were heterozygous for the frequent missense mutations involving two arginine residues in the A1 domain: p.Arg1315Cys, p.Arg1315His, p.Arg1374Cys and p.Arg1374His ( Table 6).

The laboratory phenotype of the probands heterozygous for p.Arg1315Cys and p.Arg1315His mutations was similar with low levels of VWF:Ag (median 19\%) and VWF:RCo (median $11.5 \%$ ), a decreased VWF:RCo/VWF:Ag ratio (0.66) and a normal multimeric pattern. However, the proband with the mutation p.Arg1315His was compound heterozygous with the silent mutation c.7464C $>\mathrm{T}$ detected in exon 44 , which may create a new donor splicing site ( Table 6 and Suppl. Table 3, available online at www.thrombosis-online.com). The proband's mother, who was also heterozygous for p.Arg1315His, had a type 1 VWD 
Table 5: Phenotypic and molecular data of five probands with type 2B VWD and one with PT-VWD.

\begin{tabular}{|c|c|c|c|c|c|c|c|c|c|c|}
\hline \multicolumn{11}{|c|}{ Laboratory values } \\
\hline $\begin{array}{l}\text { Probands } \\
\text { ID (n) }\end{array}$ & $\begin{array}{l}\text { Gender/ } \\
\text { age } \\
\text { (M/F year) }\end{array}$ & $\begin{array}{l}\text { Bleeding } \\
\text { Score }\end{array}$ & $\begin{array}{l}\text { FVIII:C* } \\
(\%)\end{array}$ & $\begin{array}{l}\text { VWF: } \\
\mathrm{Ag}^{*}(\%)\end{array}$ & $\begin{array}{l}\text { VWF: } \\
\text { RCo* }(\%)\end{array}$ & $\begin{array}{l}\text { VWF: } \\
\text { CB (\%) }\end{array}$ & $\begin{array}{l}\text { VWF:RCo/ } \\
\text { VWF:Ag } \\
\text { Ratio }\end{array}$ & $\begin{array}{l}\text { Platelet } \\
\mathrm{X} 10^{3} / \mu \mathrm{l}\end{array}$ & $\begin{array}{l}\text { RIPA } \\
0.5 \mathrm{mg} / \mathrm{ml}\end{array}$ & $\begin{array}{l}\text { VWF } \\
\text { Multimers }\end{array}$ \\
\hline $\begin{array}{l}\text { P26, P27, } \\
\text { P28 (3) }\end{array}$ & $\begin{array}{l}F / 41 ; F / 11 ; \\
F / 30\end{array}$ & $\begin{array}{l}20,8, \\
5\end{array}$ & $\begin{array}{l}47 \\
(23-78)\end{array}$ & $\begin{array}{l}25 \\
(20-30)\end{array}$ & $\begin{array}{l}11 \\
(10-12)\end{array}$ & $\begin{array}{l}12 \\
(9-16)\end{array}$ & $\begin{array}{l}0.44 \\
(0.40-0.50)\end{array}$ & $\begin{array}{l}79,230, \\
242\end{array}$ & $\begin{array}{l}\text { NA, E } \\
N A\end{array}$ & LHMWM \\
\hline P25 (1) & $F / 41$ & 5 & 26 & 29 & 11 & 13 & 0.38 & 15 & $E$ & LHMWM \\
\hline P50 (1) & $\mathrm{F} / 11$ & 10 & 37 & 60 & 24 & 22 & 0.35 & 130 & NA & LHMWM \\
\hline P66 (1) & $\mathrm{M} / 40$ & 1 & 101 & 122 & 47 & 50 & 0.4 & 50 & $\mathrm{E}$ & $\begin{array}{l}\text { Partial } \\
\text { LHMWM }\end{array}$ \\
\hline
\end{tabular}

phenotype with higher levels of VWF:Ag (32\%) and VWF:RCo $(26 \%)$

The missense mutations p.Arg1374Cys and p.Arg1374His were the most frequent cause of type 2M VWD in our cohort of patients. All the six probands showed a decreased VWF:RCo/ VWF:Ag ratio (median 0.3), a high VWF:CB/VWF:Ag ratio and the presence of all-size multimers, albeit with a certain degree of smearing. Two probands had the missense mutation c.4195C>T; p.Arg1399Cys associated with a mild phenotype and pronounced smearing of the multimer pattern.
The last patient (P38) was compound heterozygous for four previously undescribed missense variants. However, a family study was not conducted; therefore, which of them were in cis or in trans was unknown: p.[Val343Leu(;)Ile482Met(;)Asp1373Tyr(;)His1419Gln]. Two variants (p.Asp1373Tyr and p.His1419Gln) were clustered in the A1 domain, and the other two (p.Val343Leu and p.Ile482Met) were in the VWFpp region (D1-D2 domain). Of these four variants, only one was pathogenic (p.Asp1373Tyr), whereas the other three were benign variants. The phenotype was mild with a normal multimeric pattern ( $>$ Table 6$)$.

Table 6: Phenotypic and molecular data of 20 probands with type $2 A$ and $2 M$ VWD.

\begin{tabular}{|c|c|c|c|c|c|c|c|c|c|}
\hline \multicolumn{10}{|c|}{ Laboratory values } \\
\hline $\begin{array}{l}\text { Probands } \\
\text { ID(n) }\end{array}$ & $\begin{array}{l}\text { Gender/ } \\
\text { age } \\
\text { (M/F year) }\end{array}$ & $\begin{array}{l}\text { Bleeding } \\
\text { Score }\end{array}$ & $\begin{array}{l}\text { FVIII* } \\
(\%)\end{array}$ & $\begin{array}{l}\text { VWF: } \\
\operatorname{Ag}(\%)^{*}\end{array}$ & $\begin{array}{l}\text { VWF: } \\
\text { RCo(\%)* }\end{array}$ & $\begin{array}{l}\text { VWF:RCo/ } \\
\text { VWF:Ag } \\
\text { Ratio }\end{array}$ & $\begin{array}{l}\text { VWF: } \\
\text { CB (\%) }\end{array}$ & $\begin{array}{l}\text { RIPA } \\
1.25 \mathrm{mg} / \mathrm{ml}\end{array}$ & $\begin{array}{l}\text { Multimers/ } \\
\text { subtype }\end{array}$ \\
\hline P20 (1) & $F / 36$ & 19 & 27 & 22 & 16 & 0.7 & 10 & Decresead & LHMWM/ 2A/IIE \\
\hline P23 (1) & $F / 58$ & 15 & 22 & 31 & 7 & 0.23 & 8 & Absent & LHMWM/ $2 \mathrm{~A}$ \\
\hline P24 (1) & $M / 22$ & 10 & 23 & 22 & 3 & 0.14 & 4 & Absent & LHMWM/ $2 \mathrm{~A}$ \\
\hline P42, P43 (2) & $\mathrm{M} / 46 ; \mathrm{F} / 8$ & $14 ; 24$ & $28 ; 37$ & $21 ; 14$ & $12 ; 10$ & $0.57 ; 0.71$ & $4 ; 10$ & Decresead & LHMWM/ 2A/I \\
\hline $\begin{array}{l}\text { P44, P45, } \\
\text { P46, P47 (4) }\end{array}$ & $\begin{array}{l}F / 24 ; M / 65 \\
F / 46 ; F / 28\end{array}$ & $\begin{array}{l}12 ; 15 \\
17 ; 12\end{array}$ & $40(34-49)$ & $52(42-65)$ & $13(10-23)$ & $\begin{array}{l}0.22 \\
(0.15-0.35)\end{array}$ & $11(6-19)$ & Normal & LHMWM/ 2A/II \\
\hline P30 (1) & $F / 36$ & 7 & 48 & 16 & 11 & 0.69 & 19 & Decresead & Normal/ 2M \\
\hline P29 (1) & $F / 6$ & 3 & 24 & 22 & 14 & 0.64 & NA & NA & Smear/ 2M \\
\hline P32, P33 (2) & F/48; F76 & $9 ; 1$ & $45 ; 87$ & $70 ; 36$ & $20 ; 13$ & $0.17 ; 0.36$ & NA, 32 & NA & Smear/ 2M \\
\hline $\begin{array}{l}\text { P34, P35 } \\
\text { P36, P37 (4) }\end{array}$ & $\begin{array}{l}M / 2 ; M / 73 \\
F / 63 ; F / 31\end{array}$ & $4 ; 9 ; 3$ & $37(15-60)$ & $26(20-57)$ & $8(4-10)$ & $\begin{array}{l}0.3 \\
(0.16-0.50)\end{array}$ & $20(16-45)$ & NA & Normal/ 2M \\
\hline P39, P40 (2) & $\mathrm{F} / 42 ; \mathrm{M} / 9$ & $12 ; 7$ & $100 ; 82$ & $85 ; 55$ & $31 ; 32$ & $0.36 ; 0.58$ & $53 ; 37$ & Normal & Smear/ 2M \\
\hline P38 (1) & $M / 32$ & 7 & 51 & 31 & 19 & 0.61 & 40 & Normal & Normal/ 2M \\
\hline
\end{tabular}

* Median and range; RIPA - ristocetin-induced platelet aggregation; NA - not available; LHMWM - loss of high-molecular-weight multimers; the mutation marked in bold was not previously reported; $¥$ indicates mutation in trans. 


\begin{tabular}{|l|l|l|l|}
\hline \multicolumn{2}{|l|}{ Molecular data } & \\
\hline $\begin{array}{l}\text { Exon/ } \\
\text { Intron }\end{array}$ & $\begin{array}{l}\text { Nucleotide } \\
\text { Change }\end{array}$ & $\begin{array}{l}\text { Amino Acid } \\
\text { Change }\end{array}$ & Domain \\
\hline 28 & c.3916C>T & p.Arg1306Trp & A1 \\
\hline 28 & c.3916C>T & p.Arg1306Trp & A1 \\
\hline 20 & c.2561G $>$ A $\square$ & p.Arg854GIn & D' \\
\hline 28 & c.3946G $>$ A & p.Val1316Met & A1 \\
\hline $\begin{array}{l}\text { G. } \\
\text { GP1BA }\end{array}$ & c.751G $>$ T & $\begin{array}{l}\text { p.Asp251Tyr } \\
\text { (Asp235Tyr) }\end{array}$ \\
\hline
\end{tabular}

The knowledge of all putative mutations for every patient of this cohort permitted a broad overview of the inheritance and the combination of mutant alleles that affected each VWD type. The recessive inheritance of a majority of the mutant alleles identified in types 1, 3 and $2 \mathrm{~N}$ VWD matched the following combination: missense/null in 5/7 for severe type 1; null/null in 13/15 for type 3 and missense/missense in 11/12 for type $2 \mathrm{~N}$. As expected, type 2 VWD $(2 \mathrm{~A}, 2 \mathrm{~B}$ and $2 \mathrm{M})$ showed a dominantly inherited trait with the allelic combination wild-type/missense in $23 / 25$ for type 2 ( Tables 2-6; Figure 2).

\begin{tabular}{|c|c|c|c|}
\hline \multicolumn{4}{|c|}{ Molecular data } \\
\hline $\begin{array}{l}\text { Exon/ } \\
\text { Intron }\end{array}$ & $\begin{array}{l}\text { Nucleotide } \\
\text { change }\end{array}$ & $\begin{array}{l}\text { Amino } \\
\text { Acid Change }\end{array}$ & Domain \\
\hline 26 & c.3388T >C & p.Cys1130Arg & D3 \\
\hline 28 & c. $3814 \mathrm{~T}>\mathrm{G}$ & p.Cys1272Gly & A1 \\
\hline 28 & c.3815G>T & p.Cys1272Phe & A1 \\
\hline 28 & c. $4517 C>T$ & p.Ser1506Leu & A2 \\
\hline 28 & c. $4883 \mathrm{~T}>\mathrm{C}$ & p.lle1628Thr & A2 \\
\hline 28 & c.3944G >A & p.Arg1315His & A1 \\
\hline 44 & c.7464C >T‡ & p. $(=)$ & C3 \\
\hline 28 & c. $3943 C>T$ & p.Arg1315Cys & A1 \\
\hline 28 & c. $4120 \mathrm{C}>\mathrm{T}$ & p.Arg1374Cys & A1 \\
\hline 28 & c. $4121 \mathrm{G}>\mathrm{A}$ & p.Arg1374His & A1 \\
\hline 28 & c. $4195 C>T$ & p.Arg1399Cys & A1 \\
\hline 28 & c. $4117 \mathrm{G}>\mathrm{T}$ & p.Asp1373Tyr & A1 \\
\hline
\end{tabular}

\section{Discussion}

This study comprised a genotype-phenotype correlation in a cohort of 60 unrelated Portuguese families (92 individuals) diagnosed with types 1, 2 and 3 VWD. The VWD diagnosis based on the results of a battery of laboratory tests is often difficult, and therefore, establishing patients' disease severity and risk of bleeding becomes challenging $(6,7)$. Thus, in our diagnosis laboratory, a phenotype and genotype analysis in patients with VWD has become crucial for understanding the VWF function and great interindividual variability.

As shown in $>$ Figure 1, besides the additional mutations identified by NGS, the characteristic mutation distribution across $V W F$ was preserved in each VWD type: in type $3 \mathrm{VWD}$, the mutations were scattered throughout VWF; in severe type 1 VWD, the profile was similar, but the absence of mutations in the regions coding for the A1, A2, D' and D3 domains was evident; in type $2 \mathrm{~N}$ VWD, a mutation cluster in the region coding for the D1, D' and $\mathrm{D} 3$ domains was detected; in types $2 \mathrm{~A}, 2 \mathrm{~B}$ and $2 \mathrm{M}$ VWD, the mutation spreading was notable, but a mutation cluster in the regions coding for the A1 domain emerged. These findings are in line with previous reports $(5,31-34)$.

The group of seven probands with severe type 1 VWD showed a high degree of genetic heterogeneity with 10 different mutations, of which five (three missense mutations, one nonsense and one large deletion) have not been previously described (35\%). The in silico analysis for all three missense mutations showed that they were expected to be deleterious and disease causing (Suppl. Table 1 , available online at www.thrombosis-online.com). Five out of seven probands with mutations in regions coding for the VWF D1, $\mathrm{D} 2, \mathrm{D} 4$ and $\mathrm{C} 2-\mathrm{C} 4$ domains showed an increased FVIII:C/VWF:Ag ratio, which reflected impaired VWF secretion, possibly due to misfolding, as previously described (35).

The missense mutation p.Ala631Val has only been described in a large study of healthy controls (36) as an outlier owing to a mild type 1 VWD phenotype being presented. Nevertheless, in the present study, this mutation was found in compound heterozygosity with c.7730-4C>G, and the inheritance of both mutations was responsible for a severe phenotype (Suppl. Figure 4, available online at www.thrombosis-online.com). In addition, the compound heterozygosity of the novel missense mutation p.Ala1714Pro with c. $5170+10 \mathrm{C}>\mathrm{T}$ was associated with severe type 1 VWD. Moreover, when inherited alone, this mutation exhibited a type $2 \mathrm{M} \mathrm{VWD}$ phenotype (Suppl. Figure 4, available online at www.thrombosisonline.com). The p.Ala1714Pro mutation as well as others in the A3 domain showed an accentuated defective collagen binding with a normal multimer pattern (37).

The variants c.7730-4C>G and c. $5170+10 \mathrm{C}>\mathrm{T}$, although the low probability of a deleterious effect predicted by in silico algorithms, they seem to have had some influence on VWD phenotype in these families: the first one showed effect in autosomal recessive pattern and the second one showed a cumulative effect in a compound heterozygous state on decrease of VWF levels (Suppl. Figure 4, available online at www.thrombosis-online.com). However, the variant $c .5170+10 \mathrm{C}>\mathrm{T}$ is frequent in the studied populations, 


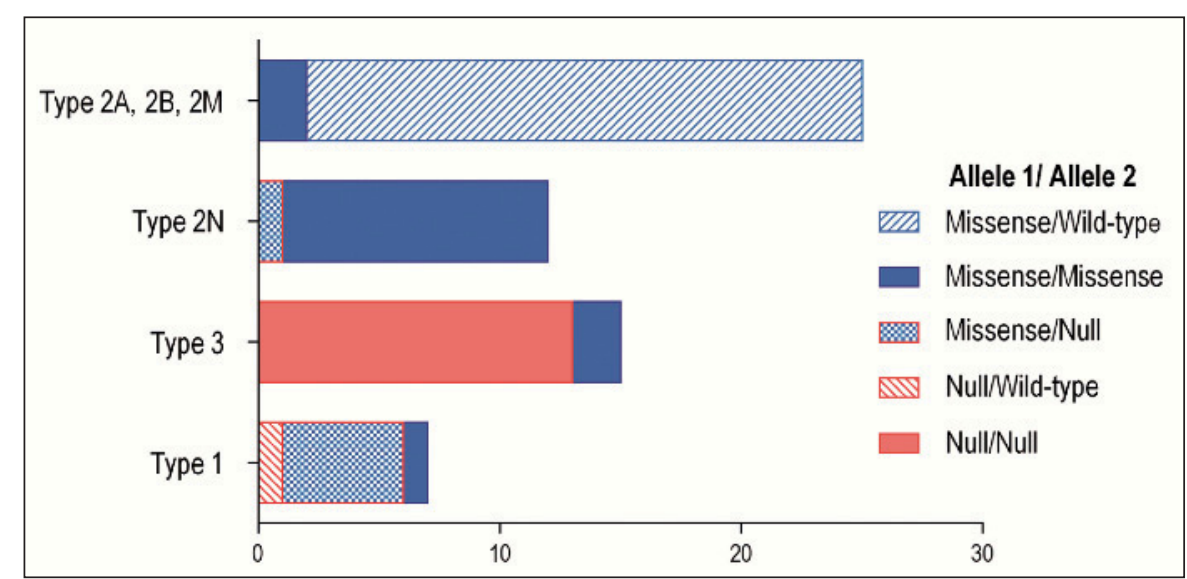

Figure 2: Mutation combination in mutated alleles in different VWD types. The main type of mutation detected in each VWD type was as follows: severe type $1 \mathrm{VWD}$, missense/null; type 3 VWD, null/null; 2N VWD, missense/missense; and type 2 VWD (2A, 2B and 2M), missense/wild type. despite the variable frequencies found in different databases (ExAC Aggregated_Populations: MAF $=0.0036$; EVS_EA: MAF $=$ 0.0046 and dbSNP: MAF $=0.0089)$ and it may be controversial to consider this a pathogenic variant. Nevertheless, it should be noted that other deep intronic changes that can only be detected by whole gene sequencing could be present and may have also influenced these phenotypes. These findings corroborate the concept that the variants' pathogenicity should be determined by the entire body of evidence in each family (18). Moreover, these likely pathogenic variants could not explain the disease; however, they seem to influence the phenotype and could behave as a pathogenic in one case and non-pathogenic in another, depending on the other co-inherited changes $(15,18)$. These data have proven the added value of practice guidelines for the evaluation of the pathogenicity of potential splice site variants. Among the six variants that were predicted as potential splice sites by in silico analysis, two were considered pathogenic, two likely pathogenic and the other two with uncertain significance (Suppl. Table 1, available online at www.thrombosis-online.com).

Therefore, these classification criteria did not replace the functional mRNA studies, the only method that can elucidate the true effect of the variation detected (38); however, this classification provided a more precise genotype-phenotype correlation.

Our cohort of type 3 VWD evidenced, as usual, two patterns of mutations: homozygous for null mutations and compound heterozygous/homozygous for missense mutations of VWF ( $>$ Figure 2). Two nonsense mutations (p.Gln1311*, p.Gln $1556^{*}$ ) and a novel small deletion (p.Val1805Glyfs ${ }^{\star} 8$ ) were expected to have the obvious deleterious mechanisms, i.e. premature termination codons and a frameshift leading to a truncated VWF protein. The homozygous p.Gln $1311^{\star}$ mutation associated with a gene conversion and initially reported in Spanish Romani families (39) was the most frequent mutation in 5/15 families, of which one was also Romani. Among these five patients, one had developed an inhibitor that has also been described in homozygous gene conversions (40). In three families studied in this cohort, obligate carriers of p.Gln $1556^{*}$ and p.Gln $1311^{*}$ were not phenotypically silent and manifested mild VWF levels and mucocutaneous bleeding symptoms, showing an inheritance pattern that is co-dominant (33) and not recessive, as described in other studies. One carrier of p.Gln $1556^{\star}$ had the variant p.Tyr1584Cys in trans without any worsening of phenotype. The small duplication p.Cys $2235 \mathrm{Argfs}^{\star} 8$ previously described in Spanish patients (10) and also repetitively found in this Portuguese study could share a common ancestral origin.

The molecular mechanisms of missense mutations in compound heterozygous state in the D3 domain (p.Tyr1146Cys) could be related to ineffective multimerisation and the subsequent defective intracellular transport and secretion with intracellular retention (41).

Finally, the p.Pro1162Leu in the homozygous state detected in a type 3 VWD proband was also found in another proband with type $2 \mathrm{~N}$ VWD ( Table 4). This controversial finding implied that p.Pro1162Leu was unlikely to be a pathogenic variant for type 3 VWD. However, the same genotype was also the only one found in a type 3 VWD Spanish family (15); therefore, it seems reasonable to expect that the p.Pro1162Leu (c.3485_3486delinsTG) variant is a part of a compound genotype that causes type $3 \mathrm{VWD}$, which has not yet been entirely explained. This possibility is in accordance with the lack of detection of molecular mechanism of type 3 VWD despite NGS improvements. The explanation for this includes deep intronic mutations that may only be identified through whole-gene sequencing and distant regulatory elements outside VWF (33).

The functional VWF:FVIIIB defect (type $2 \mathrm{~N}$ ) was mainly caused by the missense mutation p.Arg854Gln in the homozygous state in our cohort, similarly to previous studies. This is a frequent deleterious variant in Caucasian populations $(9,42)$. Nevertheless, genetic variability was introduced by compound heterozygous with novel mutations: p.Arg854Gln with the deleterious novel missense mutation p.Asp879Glu and p.Arg816Trp with the novel small deletion c.100delT (p.Arg34Aspfs ${ }^{\star} 49$ ) ( Table 4, Suppl. Table 1, available online at www.thrombosis-online.com). The ethnic variability was noted in an African proband who showed heterozygosity for the frequent polymorphism p.His817Gln in association with p.Pro1162Leu in the homozygous state. Recent multiethnic studies found that VWF missense variants in the D' and D3 domains, previously identified in European ancestry VWD probands, are more frequent in African Americans (AAs) $(36,43,44)$. 
These variants include p.His817Gln, which was strongly associated with diminished FVIII:C levels (43). The p.Pro1162Leu variant (caused by c.3485_3486delinsTG) was previously only reported in one healthy AA in the heterozygous state (36). Nevertheless, the same variant caused by a different nucleotide change (c.3485C>T) was described with polymorphic frequency in AAs (MAF $=0.262)$ $(16,17)$. Taken together these data, it could be reasonable to interpret that such different variants at the genomic level, although theoretically lead to identical amino acid change, could result in unlike consequences at the transcriptional level and to distinct deleterious effects.

This pleiotropic effect on the VWF molecule may be explained by the ethnic diversity of the VWF coding polymorphisms recently documented in large cohorts $(36,43,44)$. As previously reported, these mutations that result in low VWF antigen levels could be partially compensated by the high baseline VWF antigen levels found in Africans. They could be VWD causing only when they are confined in ethnic-specific haplotypes; in fact, only a study of large Caucasian and African cohorts could link specific VWF variants to a bleeding phenotype (44).

In agreement with previous studies, the genotype-phenotype correlation in the 25 patients with types $2 \mathrm{~A}, 2 \mathrm{~B}$ and $2 \mathrm{M}$ VWD was explained by the VWF domain affected and was therefore divided into structural and functional defects $(41,45)$.

Our type 2B VWD cohort of patients with gain-of-function mutations in the A1 domain showed two common mutations, p.Arg1306Trp and p.Val1316Met (46). In addition, we observed a highly variable platelet count with a large range (15-242 × 103 platelets/ $\mu \mathrm{l}$ ), which is consistent with previous studies ( Table 5), although only four patients had thrombocytopenia. This variability was explained by several mutations and their coding positions within the VWF A1 domain, e.g. the altered VWF GPIb-a-binding conformation (34). Although p.Arg1306Trp and p.Val1316Met are frequently correlated with thrombocytopenia, we observed some heterogeneity even in individuals with the same mutation. These data were also in agreement with the wide degree of heterogeneity of the clinical and laboratory features reported for affected members of families that have type 2B VWD (34).

Herein, we report a case of PT-VWD misdiagnosed as type $2 \mathrm{~B}$ VWD. The functional studies allowed a differential diagnosis, but molecular studies confirmed the recently described missense mutation p.Asp251Tyr (Asp235Tyr) in GP1BA (47). PT-VWD is certainly an underdiagnosed deficiency, where molecular study is a valuable diagnostic tool.

We found mutations related to all previously described type $2 \mathrm{~A}$ structural defects $(5,31,48,49)$ : i) a multimerisation defect (p.Cys1130Arg in domain D3) with the absence of large multimers and no triplet structure; ii) a change in protein folding (p.Cys1272Gly/Phe in loop of A1 domain) that causes of loss of large and intermediate multimers; iii) intracellular proteolysis of large VWF multimers (p.Ser1506Leu) with a loss of large multimers; and iv) hypersensitivity to ADAMTS13 (p.Ile1628Thr in A2 domain) with the absence of large VWF multimers and increased triplet structure ( Table 6, Suppl. Figure 3, available online at www.thrombosis-online.com).
The mutations p.Cys1130Arg and p.Ser1506Leu showed disproportionate VWF:RCo/VWF:Ag ratios, but near 0.7; the mutations p.Cys1272Gly/Phe and p.Ile1628Thr had very low ratio values, indicative of enhanced proteolysis, as previously described $(5,48)$ (Suppl. Figure 3, available online at www.thrombosis-on line.com).

Our findings in type 2M VWD patients support the well-characterised profile: mutations in the A1 domain, which are typically associated with decreased or absent RIPA and a low VWF:RCo/ VWF:Ag ratio, combined with a normal VWF:CB/VWF:Ag ratio. We identified the usual A1 domain mutation clusters, p.Arg1315Cys/His and p.Arg1374Cys/His, with the addition of the novel mutations p.Asp1373Tyr and p.His1419Gln. The VWD subtype classification of the p.Arg1374His mutation has been controversial. This mutation was described as type $2 \mathrm{~A}$ (49) or type $2 \mathrm{M}$ (50). In fact, some authors argue that the mutation is difficult to classify as type $2 \mathrm{M}$ (because of the possibly relative decrease in large VWF multimers) or any other type 2 (because of the normal banding pattern of each multimer) VWD; therefore, a classification of type 2U VWD (unclassifiable) has been suggested (51). In our study, three probands showed a multimer pattern, with the full complement of multimers, and one showed a slight decrease in the largest forms. Given the criterion of a low VWF:RCo/VWF:Ag ratio and normal VWF:CB/VWF:Ag ratio, which was evident in each of the four probands, they were classified as having type $2 \mathrm{M}$ VWD.

The same criterion was applied to the p.Arg1315Cys, p.Arg1374Cys and p.Arg1399Cys mutations that showed a smeary multimer pattern (no clear separation between individual oligomer triplets), which was particularly pronounced in two probands and two relatives with p.Arg1399Cys. This structural VWF alteration usually occurs when the responsible mutation involves cysteine residues, which may affect the disulphide bonding of VWF dimers $(9,49)$. A recent study analysed the impact of cysteine mutations that affect the carboxyl-terminal domains of VWF (52), it clearly demonstrated the importance of cysteine residues for the structural conformation and consequently multimerisation, even in patients with type 2A VWD with a smeary multimer pattern. Therefore, the smeary appearance could be associated with the alterations in VWF domains that determine whether VWD is type 2A or 2M; however, the cysteine mutations seem to be the hallmark of this multimer pattern $(9,49,52)$.

This study described, for the first time, the mutational spectrum in a cohort of Portuguese VWD patients. Overall, VWD studies highlight the high degree of variability in clinical presentation and the considerable heterogeneity of the molecular basis, which supports the great interest shown in reports from different geographic regions $(8,33,50,53,54)$. Moreover, large studies have highlighted the ethnic variability in the phenotype of many VWF missense variants $(36,43,44)$. We applied these insights to the context of familial studies to determine their usefulness for predicting individual bleeding risk. Accordingly, the genotype-phenotype correlation in each proband family with diverse VWD types was assessed to establish family overviews and elucidate phenotypic discrepancies. 
This accurate analysis was only possible because NGS has made it easier to study VWF ROIs. The NGS methodology applied in this study was the same as that used in the recently published 'Molecular and clinical profile of von Willebrand disease in Spain (PCM-EVW-ES)' project. NGS has proven to be an excellent technology that enables more rapid diagnosis with a huge economic advantage (only $70 € /$ sample, which is even cheaper than most phenotypic tests) (15). Similarly, these findings support the adjustment of our VWD diagnosis algorithm, introducing the complete sequencing of VWF (NGS) when the VWF:RCo level is $<30 \%$ or the FVIII/VWF:Ag ratio is $<0.5$.

Because of the geographical proximity of the two populations, we expected to detect the same common mutations in the patients of our cohort as for the cohort of PCM-EVW-ES (15). In fact, in type 3 VWD patients, the following were observed: the nonsense mutation p.Gln $1311^{*}$ was the most prevalent, and the missense mutation p.Pro1162Leu was also present in homozygous state. The p.Cys2235Argfs ${ }^{*} 8$ was only detected in Spanish and Portuguese patients, and interestingly, the variant p.His817Gln was also noted in association with diminished FVIII:C levels and the type $2 \mathrm{~N}$ phenotype.

Our new molecular study approach permitted the identification of 27 novel VWF mutations, with some occurring repetitively, illustrating the advantages of identifying the most prevalent mutations in a region and their correlation with the severity of bleeding phenotypes. The approach allowed us to distinguish between clinical situations that have the same symptoms with different genetic causes such as mild Haemophilia A and type 2N VWD, Bernard-Soulier syndrome and 2B VWD as well as 2B VWD and PT-

\section{What is known about this topic?}

- VWD is an inherited bleeding disorder characterised by a variable bleeding tendency and heterogeneous laboratory phenotype.

- A genotype-phenotype correlation in patients with VWD has become crucial for understanding the function and great inter-individual variability of VWF.

- With the advent of NGS, this correlation is easier to obtain because of the possibility of sequencing the entire VWF coding region for a very reasonable cost.

\section{What does this paper add?}

- Twenty-seven novel VWF mutations or potential mutations, including seven missense mutations, two nonsense mutations, two small deletions and a large deletion of exon 31, extend the mutational spectrum of VWF.

- The genotype-phenotype correlation analysis in our cohort of patients enables the unravelling of several diagnostic discrepancies and the identification of potential pleiotropic effects of mutations.

- The present study showed that NGS provides an accurate molecular analysis; however, its interplay with a detailed clinical data registry and familial studies is crucial.
VWD. In contrast, despite being a restricted study, we identified some pleiotropic mutation effects. In both situations, it was evinced that molecular studies are indispensable for an accurate diagnosis.

The molecular characterisation of VWD patients allows precise classification into the correct VWD type and the identification of carriers in familial genetic studies. This classification is particularly relevant and even mandatory for genetic counselling for type 3 VWD and, in general, for patients with higher bleeding risk. Moreover, it facilitates the evaluation of prophylactic requirements and clinical orientation, particularly in risky situations.

In conclusion, this study of 60 VWD Portuguese families will contribute to the better understanding of the molecular genetics of $V W F$-related phenotypes. NGS, in our experience, provides an effective laboratory workflow for the analysis of a single large gene, such as VWF. These findings support the idea that improving VWD diagnosis strategies will enhance clinical and laboratory approaches; thus, the most appropriate treatment for each patient can be determined.

\section{Acknowledgements}

We are grateful to the Forum Hematológico for the funding for this project. Development of VWF analysis by NGS was supported in part by a Baxter BioScience Grant (H13-000845). This work was also supported by the Spanish Ministerio de Economía y Competitividad (MINECO)-Instituto de Salud Carlos III (ISCIII) (PI1201494 and RD12/0042/0053). We like also thank to all the medical and patients who contributed to this study.

\section{Conflicts of interest}

None declared.

\section{References}

1. Sadler JE, Budde U, Eikenboom JCJ, et al. Update on the pathophysiology and classification of von Willebrand disease: a report of the Subcommittee on von Willebrand Factor. J Thromb Haemost 2006; 4: 2103-2114.

2. Batlle J, Pérez-Rodríguez A, López-Fernández MF. Classification of von Willebrand disease. In: Federici AB et al, editor. Von Willebrand Dis Basic Clin Asp 1st ed. Oxford: Wiley-Blackwell; 2011. pp. 74-85.

3. Sadler JE. von Willebrand factor assembly and secretion. J Thromb Haemost 2009; 7 (Suppl 1): 24-27.

4. Schneppenheim R. The pathophysiology of von Willebrand disease: therapeutic implications. Thromb Res 2011; 128 (Suppl): S3-7.

5. Michiels JJ, van Vliet HHDM. Dominant von Willebrand disease type 2A groups I and II due to missense mutations in the A2 domain of the von Willebrand factor gene: diagnosis and management. Acta Haematol 2009; 121: 154-166.

6. Castaman G, Hillarp A, Goodeve A. Laboratory aspects of von Willebrand disease: test repertoire and options for activity assays and genetic analysis. Haemophilia 2014; 20 (Suppl 4): 65-70.

7. Budde U, Favaloro E A. Laboratory diagnosis of von Willebrand disease: the phenotype. In: Federici AB et al, editor. Von Willebrand Dis Basic Clin Asp 1st ed. Oxford: Wiley-Blackwell; 2011. pp. 100-113.

8. Corrales I, Ramírez L, Altisent C, et al. Rapid molecular diagnosis of von Willebrand disease by direct sequencing. Detection of 12 novel putative mutations in VWF gene. Thromb Haemost 2009; 101: 570-576.

9. Goodeve AC. The genetic basis of von Willebrand disease. Blood Rev 2010; 24: 123-134.

10. Corrales I, Catarino S, Ayats J, et al. High-throughput molecular diagnosis of von Willebrand disease by next generation sequencing methods. Haematologica 2012; 97: 1003-1007. 
11. Goodeve AC. Current avenues in Von Willebrand disease research. Haematologica 2014; 8: 47-52.

12. Rodeghiero F, Castaman G, Tosetto A. How I treat von Willebrand disease. Blood 2009; 114: 1158-1165.

13. Pérez-Rodríguez A, García-Rivero A, Lourés E, et al. Autosomal dominant C1149R von Willebrand disease: Phenotypic findings and their implications. Haematologica 2009; 94: 679-686.

14. Siaka C, Rugeri L, Caron C, et al. A new ELISA assay for diagnosis of acquired von Willebrand syndrome. Haemophilia 2003; 9: 303-308.

15. Batlle J, Pérez-Rodríguez A, Corrales I, et al. Molecular and clinical profile of von Willebrand disease in Spain (PCM - EVW - ES): Proposal for a new diagnostic paradigm. Thromb Haemost 2015; 2: 1-11.

16. National Center for Biotechnology Information dbSNP (build 137). Available from: http://www.ncbi.nlm.nih.gov/snp. Accessed July 14, 2015.

17. 1000 Genomes. A deep catalog of human genetic variation. Available from: www.1000genomes.org. Accessed July 14, 2015.

18. Richards S, Aziz N, Bale S, et al. ACMG Standards and Guidelines Standards and guidelines for the interpretation of sequence variants : a joint consensus recommendation of the American College of Medical Genetics and Genomics and the Association for Molecular Pathology. Genet Med 2015; 17: 405-424.

19. Gonzalez-Garay ML. The road from next-generation sequencing to personalized medicine. Per Med 2014; 11: 523-544.

20. Jian X, Boerwinkle E, Liu X. In silico tools for splicing defect prediction: a survey from the viewpoint of end users. Genet Med 2014; 16: 497-503.

21. Wallis Y, Payne S, Mcanulty C, et al. Practice Guidelines for the Evaluation of Pathogenicity and the Reporting of Sequence Variants in Clinical Molecular Genetics. Available from: http://www.acgs.uk.com/media/774853/evaluation. Accessed September 21, 2015.

22. Mort M, Sterne-Weiler T, Li B, et al. MutPred Splice: machine learning-based prediction of exonic variants that disrupt splicing. Genome Biol 2014; 15: R19.

23. MacArthur DG, Manolio TA, Dimmock DP, et al. Guidelines for investigating causality of sequence variants in human disease. Nature 2014; 508: 469-476.

24. Exome Aggregation Consortium (ExAC), Cambridge, MA. Available from: http://exac.broadinstitute.org/. Accessed September 21, 2015.

25. Exome Variant Server, NHLBI GO Exome Sequencing Project (ESP), Seattle, WA. Available from: http://evs.gs.washington.edu/EVS/. Accessed September 21, 2015.

26. HGMD Human gene mutation database (HGMD ${ }^{\circledR}$ Professional) from BIOBASE Corporation. Available from: www.biobase-international.com/hgmd. Accessed September 21, 2015.

27. International Society on Thrombosis and Haemostasis-Scientific and Standardisation Committee of von Willebrand Factor (ISTH-SSC). VWF Online Database (VWFdb). Available from: http://www.vwf.group.shef.ac.uk/vwd.html. Accessed September 21, 2015

28. European Association for Haemophilia and Allied Disorders (EAHAD). Coagulation Factor Variant Databases. Available from: https://www.grenada.lumc.nl/ LOVD2/VWF/home.php?select_db=VWF. Accessed September 21, 2015.

29. Hemobase. Available from: http://vwf.hemobase.com/. Accessed September 21, 2015.

30. Goodeve A, Reitsma P, Mcvey J. Nomenclature of genetic variants in hemostasis. J Thromb Haemost 2011; 9: 852-855.

31. Gadisseur A, Berneman Z, Schroyens W, et al. Laboratory Diagnosis of von Willebrand Disease Type 1/2E (2A Subtype IIE), Type 1 Vicenza and Mild Type 1 Caused by Mutations in the D3, D4, B1-B3 and C1-C2 Domains of the von Willebrand Factor Gene. Acta Haematol 2009; 121: 128-138.

32. Michiels JJ, Gadisseur A, van der Planken M, et al. Laboratory and molecular characteristics of recessive von Willebrand disease type 2C (2A subtype IIC) of variable severity due to homozygous or double heterozygous mutations in the D1 and D2 domains. Acta Haematol 2009; 121: 111-118.

33. Bowman M, Tuttle A, Notley C, et al. The genetics of Canadian type 3 von Willebrand disease: Further evidence for co-dominant inheritance of mutant alleles. J Thromb Haemost 2013; 11: 512-520.

34. Federici AB, Mannucci PM, Castaman G, et al. Clinical and molecular predictors of thrombocytopenia and risk of bleeding in patients with von Willebrand disease type 2B : a cohort study of 67 patients. Blood 2011; 113: 526-534.

35. Eikenboom J, Hilbert L, Ribba AS et al. Expression of 14 von Willebrand factor mutations identified in patients with type 1 von Willebrand disease from the MCMDM-1VWD study. J Thromb Haemost 2009; 7: 1304-1312.
36. Bellissimo DB, Christopherson PA, Flood VH, et al. VWF mutations and new sequence variations identified in healthy controls are more frequent in the African-American population. Blood 2012; 119: 1-3.

37. Riddell AF, Gomez K, Millar CM, et al. Characterization of W1745C and S1783A: 2 novel mutations causing defective collagen binding in the A3 domain of von Willebrand factor. Blood 2009; 114: 3489-3496.

38. Corrales I, Ramírez L, Altisent C, et al. The study of the effect of splicing mutations in von Willebrand factor using RNA isolated from patients' platelets and leukocytes. J Thromb Haemost 2011; 9: 679-688.

39. Casaña P, Martínez F, Haya S, et al. Q1311X: a novel nonsense mutation of putative ancient origin in the von Willebrand factor gene. Br J Haematol 2000; 111: 552-555.

40. Surdhar GK. Homozygous gene conversion in von Willebrand factor gene as a cause of type 3 von Willebrand disease and predisposition to inhibitor development. Blood 2001; 98: 248-250.

41. Schneppenheim R, Budde U. von Willebrand factor: the complex molecular genetics of a multidomain and multifunctional protein. J Thromb Haemost 2011; 9 (Suppl 1): 209-215.

42. Jacquemin M. Factor VIII-von Willebrand factor binding defects in autosomal recessive von Willebrand disease type Normandy and in mild hemophilia A. New insights into factor VIII-von Willebrand factor interactions. Acta Haematol 2009; 121: 102-105.

43. Johnsen JM, Auer PL, Morrison AC, et al. Common and rare von Willebrand factor (VWF) coding variants, VWF levels, and factor VIII levels in African Americans: the NHLBI Exome Sequencing Project. Blood 2013; 122: 590-597.

44. Wang QY, Song J, Gibbs R, et al. Characterizing polymorphisms and allelic diversity of von Willebrand factor gene in the 1000 Genomes. J Thromb Haemost 2013; 11: 261-269.

45. Meyer D, Fressinaud E, Mazurier C. Clinical, laboratory, and molecular markers of type 2 von Willebrand disease. In: Federici AB, Lee CA, Berntorp EE, Lillicrap D, Montgomery RR E, editor. Von Willebrand Dis Basic Clin Asp Oxford: Wiley-Blackwell; 2011. pp. 137-147.

46. Gadisseur A, Hermans C, Berneman Z, et al. Laboratory diagnosis and molecular classification of von Willebrand disease. Acta Haematol 2009; 121: 71-84.

47. Enayat S, Ravanbod S, Rassoulzadegan M, et al. A novel D235Y mutation in the GP1BA gene enhances platelet interaction with von Willebrand factor in an Iranian family with platelet-type von Willebrand disease. Thromb Haemost 2012; 108: 946-954.

48. Woods I, Sanchez-Luceros A, Kempfer A, et al. C1272F: A novel type 2A von Willebrand's disease mutation in A1 domain; its clinical significance. Haemophilia 2012; 18: 112-116.

49. Budde U, Schneppenheim R, Eikenboom J, et al. Detailed von Willebrand factor multimer analysis in patients with von Willebrand disease in the European study, molecular and clinical markers for the diagnosis and management of type 1 von Willebrand disease (MCMDM-1VWD). J Thromb Haemost 2008; 6: 762-771.

50. Castaman G, Federici A, Tosetto A, et al. Different bleeding risk in type 2A and $2 \mathrm{M}$ von Willebrand disease: A 2-year prospective study in 107 patients. J Thromb Haemost 2012; 10: 632-638.

51. Gadisseur A, van der Planken M, Schroyens W, et al. Dominant von Willebrand disease type $2 \mathrm{M}$ and $2 \mathrm{U}$ are variable expressions of one distinct disease entity caused by loss-of-function mutations in the A1 domain of the von Willebrand factor gene. Acta Haematol 2009; 121: 145-153.

52. Yadegari H, Driesen J, Pavlova A, et al. Insights into pathological mechanisms of missense mutations in C-terminal domains of von Willebrand factor causing qualitative or quantitative von Willebrand disease. Haematologica 2013; 98: 1315-1323.

53. Hampshire DJ, Abuzenadah AM, Cartwright A, et al. Identification and characterisation of mutations associated with von Willebrand disease in a Turkish patient cohort. Thromb Haemost 2013; 110: 264-274.

54. Yadegari H, Driesen J, Pavlova A, et al. Mutation distribution in the von Willebrand factor gene related to the different von Willebrand disease (VWD) types in a cohort of VWD patients. Thromb Haemost 2012; 108: 662-671.

55. Zhou Y-F, Eng ET, Zhu J, et al. Sequence and structure relationships within von Willebrand factor. Thombosis Hemost 2012; 120: 449-458.

56. Lenting PJ, Christophe OD. von Willebrand factor biosynthesis, secretion, and clearance: connecting the far ends. Blood 2015; 125: 2019-2029. 